\title{
ON THE STRUCTURE OF SELFADJOINT TOEPLITZ OPERATORS WITH RATIONAL MATRIX SYMBOLS ${ }^{1}$
}

\author{
LEIBA RODMAN
}

\begin{abstract}
Selfadjoint Toeplitz operators with rational matrix symbols are studied using a general result concerning functions $\left(T(z)^{-1} x, y\right)$ where $T(z)$ is a polynomial family of Toeplitz operators with rational matrix symbols. It is proved that, apart from a finite number of points, these functions can be continued analytically across the boundary of the resolvent set of $T(z)$, for a dense set of $x$ 's and $y$ 's. This implies piecewise analyticity of the spectral measure $(E x, x)$ of selfadjoint Toeplitz operators with rational matrix symbol, for a dense set of $x$ 's.
\end{abstract}

1. Introduction and main result. There is a lot of knowledge available concerning the structure of selfadjoint Toeplitz operators with scalar symbols (see, e.g., $[13,16])$. However, in the case of selfadjoint Toeplitz operators with matrix symbols little is known. If the symbol of such an operator is a rational matrix function, then the spectrum consists of a finite number of closed (possibly degenerated) intervals, and the number of eigenvalues is finite (see [3]). Absolutely continuous components of selfadjoint Toeplitz operators with (not necessarily rational) matrix symbols are studied in $[\mathbf{1 7}]$.

The following theorem concerning the spectral measure of selfadjoint Toeplitz operators with rational matrix symbol is the main result of this paper. We denote by $H^{2}\left(\mathbf{C}^{n}\right)$ the Hardy space of $n$-dimensional complex vector functions $\sum_{j=0}^{\infty} \lambda^{j} x_{j}$, $x_{j} \in \mathbf{C}^{n},|\lambda|=1$ with $\sum_{j=0}^{\infty}\left\|x_{j}\right\|^{2}<\infty$. By $P\left(\mathbf{C}^{n}\right)$ we denote the linear set of $n$-dimensional vector polynomials (note that $P\left(\mathbf{C}^{n}\right)$ is dense in $H^{2}\left(\mathbf{C}^{n}\right)$ ).

THEOREM 1. Let T: $H^{2}\left(\mathbf{C}^{n}\right) \rightarrow H^{2}\left(\mathbf{C}^{n}\right)$ be a selfadjoint Toeplitz operator with rational $n \times n$ matrix symbol, and let $E_{T}(\mu),-\infty<\mu<\infty$, be the spectral measure of $T$. Then there exists a finite number of real points $\mu_{1}<\mu_{2}<\cdots<\mu_{\alpha}$ such that for every $x \in P\left(\mathbf{C}^{n}\right)$ the real function $\left(E_{T}(\mu) x, x\right)$ of $\mu \in \mathbf{R}$ is analytic in each interval $\left(\mu_{i}, \mu_{i+1}\right), i=0, \ldots, \alpha$ (we put $\left.\mu_{0}=-\infty, \mu_{\alpha+1}=\infty\right)$.

The proof of Theorem 1 will be given in $\S 3$. It is based on a general result concerning analytic continuation of functions of type $\left(T(z)^{-1} x, y\right), x, y \in P\left(\mathbf{C}^{n}\right)$, where $T(z)$ is a Toeplitz operator (not necessarily selfadjoint) with rational matrix symbol depending polynomially on the parameter $z$.

Received by the editors November 24, 1982 and, in revised form, November 30, 1983.

1980 Mathematics Subject Classification. Primary 47B35.

Key words and phrases. Selfadjoint Toeplitz operator, rational matrix symbol, singular continuous component, polynomial family of Toeplitz operators.

${ }^{1}$ Partially supported by the Basic Research Fund of the Isareli Academy of Sciences and Humanities. 
This result will be stated in the next section. Its proof in turn is based on the characterization and explicit description of left canonical factorization for certain rational matrix functions, obtained in $[\mathbf{9}, \mathbf{1 0}]$.

Note the following corollary from Theorem 1.

COROLlaRY 2. A selfadjoint Toeplitz operator $T$ with rational matrix symbol does not have a singular continuous component.

In other words, for every $x \in H^{2}\left(\mathbf{C}^{n}\right)$ the function of bounded variation $f_{T, x}(\mu)$ $=\left(E_{T}(\mu) x, x\right)$ is a sum of an absolutely continuous function and of a jump function.

Indeed, let $Q_{T}$ be the set of all $x \in H^{2}\left(\mathbf{C}^{n}\right)$ for which the function $f_{T, x}(\mu)$ does not have a singular continuous component. Theorem 1 shows that $P\left(\mathbf{C}^{n}\right) \subset Q_{T}$. Now $Q_{T}$ is a closed subspace (see, e.g., $\S \mathrm{X} .1 .2$ in $[\mathbf{1 4}]$ ); hence we obtain $Q_{T}=$ $H^{2}\left(\mathbf{C}^{n}\right)$, and Corollary 2 follows.

Thereby a conjecture concerning Toeplitz operators with matrix symbols is confirmed in the case of ratinal matrix symbols. The conjecture states that a selfadjoint Toeplitz operator with (bounded measurable) matrix symbol has no singular continuous spectrum. For scalar symbols this conjecture is true. Actually, a selfadjoint Toeplitz operator $T$ with scalar symbol is absolutely continuous (except when $T=\alpha I, \alpha \in \mathbf{R})$, see $[\mathbf{1 5}]$.

2. Polynomial families of Toeplitz operators. Let $C(\lambda, z)$ be an $n \times n$ matrix function of the form

$$
C(\lambda, z)=\left(\frac{p_{i j}(\lambda, z)}{q_{i j}(\lambda)}\right)_{i, j=1}^{n}
$$

where $p_{i j}(\lambda, z)$ is a scalar polynomial on the complex variables $\lambda, z$, and $q_{i j}(\lambda)$ is a scalar polynomial on $\lambda$ with zeros off the unit circle. For each $z \in \mathbf{C}$, consider the Toeplitz operator $T(z): H^{2}\left(\mathbf{C}^{n}\right) \rightarrow H^{2}\left(\mathbf{C}^{n}\right)$ with symbol $C(\cdot, z)$ :

$$
T(z)\left(\sum_{j=0}^{\infty} \lambda^{j} x_{j}\right)=\sum_{k=0}^{\infty}\left(\sum_{j=-\infty}^{k} C_{j}(z) x_{k-j}\right) \lambda^{k}, \quad \sum_{j=0}^{\infty} \lambda^{j} x_{j} \in H^{2}\left(\mathbf{C}^{n}\right),
$$

where $C(\lambda, z)=\sum_{j=-\infty}^{\infty} \lambda^{j} C_{j}(z)$. We shall assume that the set $\Omega_{0}=\{z \in \mathbf{C} \mid T(z)$ is invertible $\}$ is not empty. The boundary $\partial \Omega_{0}$ of $\Omega_{0}$ is a compact set having finite number of connected components (see $[\mathbf{1}, \mathbf{1 2}]$ ).

It will be convenient to represent the inverse $T(z)^{-1}, z \in \Omega_{0}$, as an infinite block matrix $\left\{t_{i j}^{-}(z)\right\}_{i, j=0}^{\infty}$ where $t_{i j}^{-}(z)$ is the $n \times n$ matrix defined by the property that

$$
\left\langle t_{i j}^{-}(z) a, b\right\rangle=\left(T(z)^{-1}\left(\lambda^{j} a\right), \lambda^{i} b\right), \quad x, y \in \mathbf{C}^{n},
$$

where $\langle\cdot, \cdot\rangle$ is the standard scalar product in $\mathbf{C}^{n}$ and $(\cdot, \cdot)$ is the scalar product in $H^{2}\left(\mathbf{C}^{n}\right)$. Clearly, the matrix functions $t_{i j}^{-}(z) ; i, j=0,1, \ldots$, are analytic in $\Omega_{0}$.

THEOREM 3. There is a finite set $K \subset \partial \Omega_{0}$ such that every function $t_{i j}^{-}(z)$; $i, j=0,1, \ldots$, admits analytic continuation from $\Omega_{0}$ across the boundary $\partial \Omega_{0}$, except maybe for poles and algebraic branch points in $K$ (the case when the same point is a pole and an algebraic branch point is not excluded).

The proof shows that a neighborhood of $\partial \Omega_{0}$ to which $t_{i j}^{-}(z)$ admits analytic continuation can be chosen to be independent on $i, j$. The number of branches of 
$t_{i j}^{-}(z)$ at a point in $K$ is bounded by a constant independent on $i, j$. However, the multiplicity of a pole of $t_{i j}^{-}(z)$ in $K$ may grow indefinitely if either $i$ or $j$ tends to infinity.

Theorem 3 implies that for every $x, y \in P\left(\mathbf{C}^{n}\right)$ the function $\left(T(z)^{-1} x, y\right)$ admits analytic continuation from $\Omega_{0}$, except maybe for poles and algebraic branch points in $K$. Actually, this property holds also for $x, y \in H^{2}\left(\mathbf{C}^{n}\right)$ which are analytic functions in a neighborhood of the closed unit disc (the proof of this fact, as well as the proof of Theorem 3, follows from formula (2) below).

In fact, we shall prove that the inverse $T(z)^{-1}$ has the following form:

$$
\begin{array}{r}
T(z)^{-1} w=q_{+}(\lambda) X(z)\left(\sum_{k=0}^{\infty} \lambda^{k}(U(z))^{k}\right) Q\left(\sum_{k=0}^{\infty} \lambda^{-k}(W(z))^{k}\right) q_{-}(\lambda) w \\
w \in H^{2}\left(\mathbf{C}^{n}\right), z \in \Omega_{0} .
\end{array}
$$

Here $q_{+}(\lambda)$ (resp. $q_{-}(\lambda)$ ) are scalar polynomials in $\lambda$ (resp. in $\lambda^{-1}$ ) with no zeros in the domain $\{|\lambda| \leq 1\}$ (resp. in the domain $\{|\lambda| \geq 1\} \cup(\infty)$ ); $Q$ is the orthogonal projector in $H^{2}\left(\mathbf{C}^{n}\right) ; X(z), U(z)$ and $W(z)$ are analytic matrix functions in $z \in \Omega_{0}$ which admit analytic continuation across the boundary $\partial \Omega_{0}$ except possibly for a finite number of poles and algebraic branch points on $\partial \Omega_{0}$ (the case when the same point is a pole and an algebraic branch point, is not excluded), and $\|U(z)\|<1$, $\|W(z)\|<1$ for $z \in \Omega_{0}$ (so the power series in (2) converge). The norms of matrices are understood as operator norms with the euclidean norm in $\mathbf{C}^{n}$. The matrix function $X(z)$ has the additional property that $X(z)$ is invertible for $z \in \Omega_{0}$.

Representation (2) implies Theorem 3 immediately. Indeed, for $a, b \in \mathbf{C}^{n}$ the expression $\left(T(z)^{-1}\left(\lambda^{j} a\right), \lambda^{j} b\right)$ contains only a finite number of powers $(U(z))^{k}$, $(W(z))^{k}$.

So, in order to prove Theorem 3 we have to prove formula (2). This will be done in $\S 4$.

3. Proof of Theorem 1. By Theorem 3, there is a finite number of real points $\mu_{1}<\mu_{2}<\cdots<\mu_{\beta}$ such that for every $w \in P\left(\mathbf{C}^{n}\right)$ the function $\left((z-T)^{-1} w, w\right)$ admits analytic continuation across the real line in every interval which does not contain any point $\mu_{i}$.

Denote by $f_{w}^{+}(z)$ (resp. $\left.f_{w}^{-}(z)\right)$ the analytic continuation of $\left((z-T)^{-1} w, w\right)$ across the real line from the upper (resp. lower) halfplane.

The following formula holds for any real $\nu_{1}<\nu_{2}$ which are different from the eigenvalues of $T$ (see [19, Chapter XI, §9]):

$$
\begin{gathered}
\lim _{\nu \downarrow 0}\left[\int_{\nu_{1}}^{\nu_{2}}\left(((u-i v) I-T)^{-1} x, x\right) d u+\int_{\nu_{2}}^{\nu_{1}}\left(((u+i v) I-T)^{-1} x, x\right) d u\right] \\
=2 \pi i\left[\left(E_{T}\left(\nu_{2}\right) x, x\right)-\left(E_{T}\left(\nu_{1}\right) x, x\right)\right], \quad x \in H^{2}\left(\mathbf{C}^{n}\right) .
\end{gathered}
$$

Choose $\nu_{1}, \nu_{2}$ in such a way that the interval $\left[\nu_{1}, \nu_{2}\right]$ does not contain any point $\mu_{i}, \mathbf{1} \leq i \leq \beta$, and does not contain any eigenvalue of $T$ (by $[3]$ the number of eigenvalues of $T$ is finite). Then for $x \in H_{0}^{2}\left(\mathbf{C}^{n}\right)$ we have

$$
\int_{\nu_{2}}^{\nu_{1}} f_{x}^{-}(u) d u+\int_{\nu_{2}}^{\nu_{1}} f_{x}^{+}(u) d u=2 i\left[\left(E_{T}\left(\nu_{2}\right) x, x\right)-\left(E_{T}\left(\nu_{1}\right) x, x\right)\right],
$$

and since $f_{x}^{ \pm}(u)$ are analytic in $\left[\nu_{1}, \nu_{2}\right]$, the same is true for $\left(E_{T}(u) x, x\right)$. This completes the proof of Theorem 1 . 
4. Proof of formula (2). We recall first some relevant facts concerning Toeplitz operators. Consider the function

$$
C(\lambda)=\lambda^{-1} C_{-1}+C_{0}+\lambda C_{1},
$$

where $C_{i}, i=0, \pm 1$, are $n \times n$ complex matrices, and let $T$ be the Toeplitz operator with the symbol $C(\lambda)$. We shall assume that $\operatorname{det} C(\lambda) \neq 0$ for $\lambda$ belonging to the unit circle $\Gamma_{0}$. Write

$$
C(\lambda)^{-1}=\sum_{j=-\infty}^{\infty} \lambda^{j} D_{j}, \quad \lambda \in \Gamma_{0} .
$$

LEMMA 4. T is invertible if and only if the matrix $\left[\begin{array}{ll}D_{1} & D_{0}\end{array}\right]$ is right invertible and the matrix $\left[\begin{array}{l}D_{0} \\ D_{-1}\end{array}\right]$ is left invertible. In this case

$$
T^{-1}=T_{+} \cdot T_{-},
$$

where $T_{+}$(resp. $T_{-}$) is the Toeplitz operator with the symbol $C_{+}(\lambda)^{-1}$ (resp. $\left.C_{-}(\lambda)^{-1}\right)$, and the functions $C_{ \pm}(\lambda)$ are given by the following formulas:

$$
\begin{gathered}
C_{-}(\lambda)=I-\lambda^{-1} W, \\
W=\left[\begin{array}{c}
D_{0} \\
D_{-1}
\end{array}\right]^{-1}\left[\begin{array}{l}
D_{-1} \\
D_{-2}
\end{array}\right]
\end{gathered}
$$

and

$$
\left[\begin{array}{c}
D_{0} \\
D_{-1}
\end{array}\right]^{-1} \quad \text { is any left inverse of } \quad\left[\begin{array}{c}
D_{0} \\
D_{-1}
\end{array}\right]
$$

$$
C_{+}(\lambda)=V\left(I+\lambda V^{-1} C_{1}\right)
$$

where $V \stackrel{\text { def }}{=} C_{0}+C_{1} W$ is invertible.

PROOF. The proof is obtained essentially by putting together known facts. A general result proved in [7] implies that $T$ is invertible if and only if $\operatorname{det} C(\lambda) \neq 0$ for $\lambda$ belonging to the unit circle $\Gamma_{0}$, and the function $C(\lambda)$ admits a right canonical factorization:

$$
C(\lambda)=\tilde{C}_{-}(\lambda) \cdot \tilde{C}_{+}(\lambda), \quad \lambda \in \Gamma_{0},
$$

where $\tilde{C}_{-}(\lambda)$ (resp. $\tilde{C}_{+}(\lambda)$ ) is an invertible continuous function of $\Gamma_{0}$ which admits analytic and invertible continuation to the domain $\{|\lambda|>1\} \cup\{\infty\}$ (resp. to the domain $\{|\lambda|<1\}$; moreover, in this case $T^{-1}=T_{+} \cdot T_{-}$, where $T_{+}$(resp. $\left.T_{-}\right)$is the Toeplitz operator with the symbol $\tilde{C}_{+}(\lambda)^{-1}$ (resp. $\left.\tilde{C}_{-}(\lambda)^{-1}\right)$. By Corollary 3.29 in $[\mathbf{1 0}]$, the existence of right canonical factorization of $C(\lambda)$ is equivalent to right invertibility of the matrix $\left[\begin{array}{ll}D_{1} & D_{0}\end{array}\right]$ and left invertibility of the matrix $\left[\begin{array}{c}D_{0} \\ D_{-1}\end{array}\right]$.

Using the results from $\S 3$ in $[\mathbf{9}]$ it is easy to show that the factor $\tilde{C}_{+}$(resp. $\tilde{C}_{-}$) in the right canonical factorization (7) can be chosen to coincide with $C_{+}$(resp. $C_{-}$) given by formula (6) (resp. (5)).

Recently I. Gohberg and L. Lerer [8] proved Lemma 4 directly (without reference to a right canonical factorization of $C(\lambda)$ ), with a formula for $T^{-1}$ which does not involve $C_{ \pm}$. 
We start now to prove formula (2). Consider first the case when

$$
C(\lambda, z)=\sum_{j=-m}^{m} \lambda^{j} C_{j}(z), \quad \lambda \in \mathbf{C},
$$

where $C_{j}(z)(j=0, \pm 1, \ldots, \pm m)$ are $n \times n$ matrix polynomials. We shall assume $m=1$ (otherwise consider the function

$$
\begin{array}{r}
{\left[\begin{array}{cccc}
C_{m}(z) & C_{m-1}(z) & \ldots & C_{1}(z) \\
0 & C_{m}(z) & \ldots & C_{2}(z) \\
\vdots & \vdots & & \vdots \\
0 & 0 & \ldots & C_{m}(z)
\end{array}\right]+\left[\begin{array}{cccc}
C_{0}(z) & C_{-1}(z) & \ldots & C_{-m+1}(z) \\
C_{1}(z) & C_{0}(z) & \ldots & C_{-m+2}(z) \\
\vdots & \vdots & & \vdots \\
C_{m-1}(z) & C_{m-2}(z) & \ldots & C_{0}(z)
\end{array}\right]} \\
+\lambda^{-1}\left[\begin{array}{cccc}
C_{-m}(z) & 0 & \ldots & 0 \\
C_{-m+1}(z) & C_{-m}(z) & \ldots & 0 \\
\vdots & \vdots & & \vdots \\
C_{-1}(z) & C_{-2}(z) & \ldots & C_{-m}(z)
\end{array}\right]
\end{array}
$$

in place of $C(\lambda, z))$. This reduction to the case $m=1$ was originated and studied in [4]; see also [2].

Write $C(\lambda, z)=\sum_{j=-\infty}^{\infty} \lambda^{j} D_{j}(z), \lambda \in \Gamma_{0}$; then for $z \in \Omega_{0}$ the matrix

$$
\left[\begin{array}{ll}
D_{1}(z) & D_{0}(z)
\end{array}\right] \quad\left(\operatorname{resp} .\left[\begin{array}{c}
D_{0}(z) \\
D_{-1}(z)
\end{array}\right]\right)
$$

is right (resp. left) invertible and the matrix $C(\lambda, z)$ admits the right canonical factorization (4) with $C_{-}(\lambda, z)$ and $C_{+}(\lambda, z)$ given by (6) and (7) respectively, where $W=W(z), V=V(z)$ are functions of $z$. Taking into account that $C_{ \pm}(\lambda, z)$ are also analytic in the annulus $\rho<|\lambda|<\rho^{-1}$, we obtain that $\|W(z)\|<1$, $\left\|V(z)^{-1} C_{1}(z)\right\|<1$ (for $z \in \Omega_{0}$ ) and

$$
\begin{gathered}
\left(C_{-}(\lambda, z)\right)^{-1}=\sum_{j=0}^{\infty} \lambda^{j}(W(z))^{j}, \\
\left(C_{+}(\lambda, z)\right)^{-1}=(V(z))^{-1} \sum_{j=0}^{\infty}(-1)^{j} \lambda^{j}\left(C_{1}(z) V(z)\right)^{-j} .
\end{gathered}
$$

So formula (2) (with $q_{+}(\lambda) \equiv q_{-}(\lambda) \equiv 1, U(z)=-\left(C_{1}(z) V(z)\right)^{-1}, X(z)=V(z)^{-1}$ ) follows from (5) provided the functions $V(z)^{-1}$ and $W(z)$ have analytic continuations across $\Omega_{0}$ with the desired properties. To prove that, consider first the function

$$
D_{j}(z)=\frac{1}{2 \pi i} \int_{\Gamma_{0}} \lambda^{-j-1} C(\lambda, z)^{-1} d \lambda, \quad z \in \Omega_{0} .
$$

A fixed entry of $D_{j}(z)$ has the form

$$
\gamma(z)=\frac{1}{2 \pi i} \int_{\Gamma_{0}} p(\lambda, z)(q(\lambda, z))^{-1} d \lambda,
$$

where $p(\lambda, z)$ and $q(\lambda, z)$ are polynomials on $\lambda$ and $z$. It is not difficult to see (e.g., Lemma 2.4 in $[1])$ that $\gamma(z)$ can be continued analytically across the boundary $\partial \Omega_{0}$ 
of $\Omega_{0}$ except for at most finite number of poles and algebraic branch points. So the same is true for each $D_{j}(z), j=0, \pm 1, \pm 2, \ldots$.

Let $\Xi \subset \mathbf{C}$ be a neighborhood of $\bar{\Omega}_{0}$ where this analytic continuation takes place for $D_{0}(z), D_{-1}(z)$, and $D_{-2}(z)$. Since

$$
\left[\begin{array}{c}
D_{0}(z) \\
D_{-1}(z)
\end{array}\right]
$$

is left invertible for $z \in \Omega_{0}$, it follows from the results of [18] (see also Lemma 3.1 in $[\mathbf{1 1}])$ that there exists a left inverse of

$$
\left[\begin{array}{c}
D_{0}(z) \\
D_{-1}(z)
\end{array}\right]
$$

which is analytic in $\Xi$ except for a finite number of poles and algebriac branch points in $\partial \Omega_{0}$, and except for at most countable set of poles in $\Xi \backslash \Omega$, whose limit points (of any) are on the boundary of $\Xi$. Taking this left inverse in the formula for $W(z)$ we obtain that $W(z)$ has the desired analytic continuation. Since $V(z)$ is invertible for $z \in \Omega_{0}$, by an analogous argument it is seen that $V(z)^{-1}$ has also the desired analytic continuation.

So the formula (2) is proved for $C(\lambda, z)$ of the form (8). The general $C(\lambda, z)$ can be reduced to the form (8) by the following argument borrowed from $[\mathbf{3}]$. For $C(\lambda, z)$ given by $(1)$, let $q(\lambda)=\prod_{i, j} q_{i j}(\lambda)$, and let $q(\lambda)=q_{+}(\lambda) \lambda^{k} q_{-}(\lambda)$, where $q_{+}(\lambda)$ (resp. $q_{-}(\lambda)$ ) is a rational function without poles and zeros in the domain $\{|\lambda| \leq 1\}$ (resp. $\{|\lambda| \geq 1\} \cup\{\infty\}$ ). Now formula (2) for the Toeplitz operator with the symbol $C(\lambda, z)$ follows from the already proved formula (2) for the Toeplitz operator with the symbol $q(\lambda) \lambda^{-k} C(\lambda, z)$.

5. Examples. We give here examples illustrating the main results.

EXAMPLE 1. Let $T$ be the Toeplitz operator with $2 n \times 2 n$ matrix symbol

$$
C(\lambda)=\left[\begin{array}{cc}
0 & A(\lambda) \\
A(\lambda)^{-1} & 0
\end{array}\right]
$$

where $A(\lambda)$ is an $n \times n$ rational matrix function which takes unitary values on the unit circle. As for each $\lambda \in \Gamma_{0}$ the matrix $C(\lambda)$ has fixed eigenvalues 1 and -1 (each with multiplicity $n$ ), it follows from the results of $[\mathbf{1 7}]$ that $T$ is purely singular. Now Corollary 2 shows that $T$ does not have a singular continuous component, so there exists an orthonormal basis in $H^{2}\left(\mathbf{C}^{2 n}\right)$ formed by eigenvectors of $T$.

EXAMPLE 2. Let $T$ be the Toeplitz operator with matrix symbol $C(\lambda)=$ $\lambda^{-1} C_{-1}+C_{0}+\lambda C_{1}$, where $C_{-1}=C_{1}^{*}$ are $n \times n$ matrices such that $0 \notin\left\{\left(C_{1} z, z\right) \mid\right.$ $\|z\|=1\}$ (in particular, $C_{-1}$ and $C_{1}$ are invertible). It turns out that $T$ is absolutely continuous. Indeed, in view of Corollary 2 we have to show that $T$ does not have eigenvectors. As the operator $T-z I, z \in \mathbf{R}$, has the same structure as $T$, it will suffice to prove $\operatorname{ker} T=(0)$. Let

$$
X=\left[\begin{array}{ll}
I & 0
\end{array}\right], \quad K=\left[\begin{array}{cc}
0 & I \\
-C_{-1}^{-1} C_{1} & -C_{-1}^{-1} C_{0}
\end{array}\right]
$$

be matrices of sizes $n \times 2 n$ and $2 n \times 2 n$, respectively, and denote by $\mathcal{L}$ the maximal $K$-invariant subspace with $\sigma\left(\left.K\right|_{\mathcal{L}}\right)$ in the open unit disc. The general form of a 
vector $x=\sum_{j=0}^{\infty} \lambda^{j} x_{j} \in H^{2}\left(\mathbf{C}^{n}\right)$ satisfying $T x=0$ is $x_{j}=X K^{j} y_{0}, j=0,1, \ldots$, where $y_{0} \in \mathcal{L}$ is such that $C_{0} X y_{0}+C_{-1} X K y_{0}=0$ (see [3], also $\S 2.6$ in [5]). Letting $y_{1}=K^{-1} y_{0}$ and using the easily verified equality

$$
C_{1} \dot{X}+C_{0} X K+C_{-1} X K^{2}=0
$$

we obtain $C_{1} X y_{1}=0$, or $X y_{1}=0$. Now to show that $x=0$ it is sufficient to verify that the only vector $y_{1} \in \mathcal{L}$ for which $X y_{1}=0$ is $y_{1}=0$. To this end note that $K^{*} B K=B$ for the hermitian matrix

$$
B=i\left[\begin{array}{cc}
0 & C_{-1} \\
-C_{1} & 0
\end{array}\right]
$$

(see $\S 2.4$ in $[6])$. But then $\left(B z_{1}, z_{2}\right)=0$ for every $z_{1}, z_{2} \in \mathcal{L}$ (see, c.g., Chapter 2 in [6]). If $y_{1} \in \mathcal{L}$ is such that $X y_{1}=0$, i.e. $y_{1}=\left[\begin{array}{l}0 \\ z\end{array}\right], z \in \mathbf{C}^{n}$, then taking $z_{2}=y_{1}$ and $z_{1}=K y_{1}$ we obtain

$$
0=\left(B z_{1}, z_{2}\right)=-i\left(C_{1} z, z\right)
$$

which implies $z=0$.

Note that the eigenvalues of $C(\lambda)$, as functions of $\lambda$, are not constant (because of the invertibility of $C_{1}$ ). The absolutely continuous components of Toeplitz operators whose matrix symbols have nonconstant eigenvalues have been studied in [17].

ACKNOWLEDGEMENT. I wish to thank Professor K. Clancey for fruitful discussions concerning the subject of this paper.

\section{REFERENCES}

1. E. Azoff, K. Clancey and I. Gohberg, On the spectra of finite dimensional perturbations of matrix multiplication operators, Manuscripta Math. 30 (1980), 351-360.

2. K. Clancey and I. Gohberg, Factorizations of matrix functions and singular integral operators, Birkhauser, Basel, Boston and Stuttgart, 1981.

3. K. Clancey and L. Rodman, Eigenspaces of families of Toeplitz operators with rational matrix symbols, Manuscripta Math. 45 (1983), 1-12.

4. I. C. Gohberg and I. A. Feldman, The indices of multiple extensions of matrix functions, Bul. Akad. Stiince RRS Moldoven 8 (1967), 76-80.

5. I. Gohberg, P. Lancaster and L. Rodman, Matrix polynomials, Academic Press, New York, 1982.

6. __ Matrices and indefinite scalar products, Birkhauser, Basel, Boston and Stuttgart, 1983.

7. I. C. Gohberg and J. Leiterer, General theorems on canonical factorization of operator-functions relative to a contour, Mat. Issled 7 (1972), vyp. 3 (25), 87-134. (Russian)

8. I. Gohberg and L. Lerer, in preparation.

9. I. Gohberg, L. Lerer and L. Rodman, On canonical factorization of operator polymomials, spectral divisors and Toeplitz matrices, Integral Equations and Operator Theory 1 (1978), 176-214.

10. Stable factorization of operator polymomials. II. Main results and applications to Toeplitz operators, J. Math. Anal. Appl. 75 (1980), 1-40.

11 I. Gohberg and L. Rodman, Analytic matrix functions with prescribed local data, J. Analyse Math. 40 (1981), 90-128.

12. G. Heinig, On the inversion and on the spectrum of Weiner-Hopf matrix operators, Math. USSR-Sb. 20 (1973), 267-281.

13. P. S. Ismagilov, On the spectrum of Toeplitz matrices, Dokl. Akad. Nauk SSSR 149 (1963), 769-772; English transl., Soviet Math. 4 (1963), 462-465.

14. T. Kato, Perturbation theory for linear operators, 2nd ed., Springer-Verlag, Berlin, Heidelberg and New York, 1976.

15. M. Rosenblum, The absolute continuity of Toeplitz matrices, Pacific J. Math. 10 (1960), 987-996. 
16. _ A concrete spectral theory for self-adjoint Toeplitz operators, Amer. J. Math. 87 (1965), 709-718.

17. J. Rovnyak, The absolutely continuous component of a selfadjoint Toeplitz operator, Indiana Univ. Math. J. 21 (1972), 751-757.

18. Yu. L. Shmuljan, Finite dimensional operators depending analytically on a parameter, Ukrain. Math. Z. 9 (1957), 195-204.

19. K. Yosida, Functional analysis, Springer-Verlag, Berlin, Göttingen and Heidelberg, 1965.

School of Mathematical Sciences, Tel-Aviv University, Tel-Aviv, Ramat AVIV, ISRAEL 